\title{
A REVIEW ON NANOSPONGES: A BOON TO TARGETED DRUG DELIVERY FOR ANTICANCER DRUG
}

\author{
NIKITA SEHGAL*, VISHAL GUPTA N, SANDEEP KANNA
}

Department of Pharmaceutics, JSS College of Pharmacy, JSS Academy of Higher Education and Research, Mysore, Karnataka, India. Email: sehgal.nikita28@gmail.com

Received: 18 March 2019, Revised and Accepted: 01 May 2019

\begin{abstract}
In recent decades, the rise in the investigation of new drugs had made health-care system expensive compared to conventional drug delivery systems and techniques. The present drug delivery systems have become highly productive and are growing fast. Majority of the anticancer agent has low water solubility resulting in multistep synthetic routes that require higher selectivity and specificity that can cause difficulty in the development of the formulation. Nanosponges (NSs) are branched cyclodextrin (CD) polymeric systems which have proven to be a boon in the pharmaceutical and biomedical fields. Different kinds of NSs based on different types of CDs and crosslinkers are used for developing of new drug formulations from the past few years for various applications in health care. Nanotechnology has overcome the issues regarding the drug solubility, stability, and other parameters and has attained success in achieving of sustained release, increased activity, improved permeability, delivery of nucleoprotein, the stimuli-responsive release of the drug, and improved drug bioavailability. There is a huge eruption of research on NSs for cancer treatment. Multiple anticancer moieties have been developed, taking into account the pharmacological and physicochemical perspective of the drug to develop a NS formulation. Our target in this review is to catch an efficient and far-reaching NSs for malignancy cancer treatment announced until now. This survey will give a perfect stage for providing details for researchers taking a shot at using new polymers for improving the treatment of the disease using nanotechnology. The present article provides details regarding antineoplastic molecules and provides ideas on CD-based NSs specifically using curcumin, tamoxifen, resveratrol, quercetin, oxygen-NSs, temozolomide, doxorubicin, and 5-fluorouracil (5-FU), and erlotinib (ETB) glutathione.
\end{abstract}

Keywords: Nanosponges, Cyclodextrin, Antineoplastic molecules.

(C) 2019 The Authors. Published by Innovare Academic Sciences Pvt Ltd. This is an open access article under the CC BY license (http://creativecommons. org/licenses/by/4. 0/) DOI: http://dx.doi.org/10.22159/ajpcr.2019.v12i7.33118

\section{INTRODUCTION}

In recent decades, the rise in the investigation of new drugs had made health-care system expensive compared to conventional drug delivery systems and techniques. The present drug delivery systems have become highly productive and are growing fast. Majority of the anticancer agent has low water solubility resulting in multistep synthetic routes that require higher selectivity and specificity, that can cause difficulty in the development of the formulation. For the optimization of therapy and cost-effective treatment, huge research is carried on drug delivery systems. Delivery of these antineoplastic agents is tough using conventional techniques as it causes the inconvenient release of active ingredient for an extended period of time and shorter duration of action which led to acute side effects and produced a toxic effect on healthy tissues. Moreover, the development of drug resistance can further reduce the efficacy of conventional drugs and most molecules are affected by degradation in the gastrointestinal tract. Due to this, the advanced targeted drug delivery systems have gained prime importance in these days [1-5].

Nanomedicine and nanotechnology landscape is evolving post haste. Nanotechnology allows control and sustained release of the drug, refinement of the physical and chemical properties of drugs producing ameliorate pharmacokinetics and biodistribution profiles without affecting the efficacy and with reduced side effects thus provides promising nanoparticle as flexible drug delivery vehicle. This technology ameliorates the therapeutic index of many compounds, extensively in case anticancer drugs, where insufficient concentration in tumor site and high toxicity of drug are the prime reason for narrow efficacy of the drug [6-9].

Nanoparticles have potential to penetrate the cells that enable intracellular accumulation of their load, which was shown for the first time by Couvreur, either by active or passive targeting, even it allows drug delivery to the specific site and tissue [10-13]. Cancerous tissue offer enhanced permeability and retention (EPR) effect as they do not belong to the reticuloendothelial system $[14,15]$. By the EPR, passive targeting of the nanoparticles had evaded through tumor vessel leading to flawed architecture. Tumor is said to have a larger pore size (380-780) compared to healthy organs. Hence, in this type of targeting, the particle size of the system is of prime concern as tumor tissue accumulation by EPR relies on extravasation through pores on hyperpermeable tumor vasculature $[13,16]$.

In the case of active targeting, modification of nanoparticles surface using specific ligands such as sugar, antibiotics, and peptides helps to recognize and bind with specific cell receptor [17]. Due to this, research is focused on using nanotechnology for the use of a different chemotherapeutic agent. Nanocarriers include liposomes, dendrimers, gold nanoparticle, and a magnetic nanoparticle [4]. At present, Doxil, Abraxane, and Daunoxome are the nanoparticle medicines available in market approved by the Food and Drug Administration for treating cancer [19].

Another procedure that specialists have found against the tumor is focusing on its lower $\mathrm{pH}$ and increment the temperature of the tumor due to an explicit moiety in the polymer structure which acts as a receptive for the outside improvements, for example, separation of the carboxylic assembly and the redox-responsive group may encourage confined drug delivery. This method of conveying boosts the drug delivery utilizing nanoparticle helps in focusing on the tumor [20].

Thus, to conquer incapability of present and forthcoming antineoplastic agents and also to the delivery drug with optimum efficacy and minimal side effects, there is a need for novel nanostructured molecules and nano techniques consisting of carrier systems. 
Modern search focuses on the development of novel drug delivery systems such as nanoporous and mesoporous (organic or inorganic based NSs) that have potential application in drug delivery and nanotherapeutics. Due to the toxicity caused by inorganic systems, research prominence has been laid on organic systems [21]. About decades ago, researchers have developed CD-based NSs to overcome the limitation of drug delivery and cancer therapeutics [3]. The goal of the present review article is to illustrate the development and application of CD-based NSs for cancer therapy.

CD NSs are formed from various organic or inorganic materials such as titanium or another metal oxide, silicon particle, and carbon coated metallic NSs. CD-based NSs were initially used for purification of water, because at low concentration also it can strongly bind to organic molecules and removes them from the water. However, now they have been used and explored in the field of pharmaceuticals and biomedical sciences such as to increase solubility, stability, bioavailability, modulation of drug release, delivery of protein, cosmetics carrier, and diagnostic.

NSs belongs to hyper crosslinked polymer based colloidal solid nanoparticles with a nanosized cavity. The crosslinkers, i.e. small molecules in a mixture of polymer solution act like tiny grappling hooks which leads to the formation of sphere-shaped particles with cavities in which drug molecules are incorporated. As it is biodegradable polyester, breaks down gradually in the body. Due to this NSs, delivery of the drug in a predictable manner with reduced side effects. They provide succours in case of hydrophobic drug exceedingly for an anticancer drug, as NSs are water soluble. Another unique property that makes NSs a choice of the delivery system is by varying the proportion of crosslinker to the polymer; any size of NSs can be formulated.

\section{The advantage of NS based delivery}

1. It provides relentless action up to $24 \mathrm{~h}$.

2. A drug that is entrapped in the polymeric cage provides sustained release with lesser side effects and can withstand temperature up to $300^{\circ} \mathrm{C}$.

3. Better solubility, stability, bioavailability, flexibility, and gracefulness.

4. As it converts liquids to powders, it offers a higher degree of material processing

5. Provides protection from light or degradation, also used in the topical delivery system.

6. It can be used as a carrier for gases like oxygen and carbon dioxide, and in the case for many diseases, it provides oxygen to hypoxic tissues.

7. Used as unpleasant taste masking.

8. NSs formulation is stable up to $130^{\circ} \mathrm{C}$ and in the $\mathrm{pH}$ range of $1-11$.

9. It enables target drug delivery as it has the ability to link with a various functional group, which can be further enhanced by means of chemical linkers, even the external magnetic field can be also be applied for target drug delivery, by incorporating magnetic properties into NSs.

10. NSs give clear to milky colloidal suspension in aqueous media, and its easy to regenerate by means of solvent extraction, thermal desorption using ultrasound.

\section{METHOD OF PREPARATION OF CD-BASED NSS}

\section{Techniques used in synthesis to prepare CD-based NSs}

1. Solvent technique

2. Melt technique

3. Ultrasound-assisted synthesis

4. Microwave-assisted synthesis.

\section{Solvent method}

In this procedure, crosslinkers are solubilized in solvents such as dimethylsulfoxide or dimethylformamide and on another side, the polar aprotic solvent is used to treat the polymer, and this solution is transferred to the above-prepared solution cross. The temperature was kept in the range of $10^{\circ} \mathrm{C}$ to the reflux temperature of solvent for $1-48 \mathrm{~h}$.
To the obtained product, the cooled solution is added and transferred to large double distilled water. Filtration is done to obtain the final product under vacuum. It is also possible to reduce the size of the obtained NSs by providing high-pressure homogenization. To avoid degradation and aggregation of the product, it is stored in the refrigerator at $4^{\circ} \mathrm{C}$.

\section{Melt technique}

In this technique, crosslinkers are allowed to melt with CDs and all other ingredients are homogenized at a temperature up to $100^{\circ} \mathrm{C}$ and magnetic stirring is done for $5 \mathrm{~h}$. This above solution is allowed to cool and continuously washed to remove unreacted excipients and byproduct that is formed during the reaction.

\section{Ultrasound-assisted synthesis}

At initial stages, under sonication, the polymer is treated with crosslinkers without adding solvent. In this procedure, $\beta-C D$ and diphenyl carbonate are blended in a suitable container, and this container is then transferred to previously filled with water in an ultrasound bath at $90^{\circ} \mathrm{C}$ for $5 \mathrm{~h}$, which is further crystallized and purified as mentioned in solvent method and melt method. This method can be put back by a process involving like high energy input using probe sonication. This method does not require the use of organic solvents, which makes it a method of choice for the individual.

\section{Microwave-assisted synthesis}

This is the easiest method for NS preparation, which offers a greater advantage over another method by having a higher degree of crystallinity and also provides four folds reduction in reaction time and uniform particle size distribution. By applying all these techniques above, NSs can be synthesized, crystallized, and purified. Out of which purification of NSs in the most crucial thing as the by-products that are formed can cause toxicity. By-products are of various natures, chemical structure and are formed depending on various crosslinkers used.

\section{NSs as anticancer agent}

\section{Delivery of CD-based NSs}

Tamoxifen

Tamoxifen comes under the class of drugs called selective estrogen receptor modulators, which have both estrogenic and antiestrogenic effect and is used to treat breast cancer in pre- and post-menopausal women [22]. However, it has several side effect, which is also life- threatening such as endometrial carcinoma, metastatic tumor, venous thrombosis, and pulmonary emboli, which are suspected to be dose dependent to necessitate the development of prolonged release formulation of tamoxifen to reduce side effects [23,24]. Apart from this, it has very low aqueous solubility, which limits its therapeutic action that again becomes a challenge in the process of developing the formulation [25-29]. Broad research has been completed on tamoxifen delivery utilizing novel nanocarriers to overcome the limitation related to the delivery of tamoxifen to the site [33,34]. Torne et al. developed $\beta$-CD- based NSs for oral delivery of tamoxifen using different cross- linking densities ( $\mathrm{F} 1_{1: 2}, \mathrm{~F} 2_{1: 4}$, and $\left.\mathrm{F} 3_{1: 8}\right)$. Out of which, formulation $\mathrm{F} 2_{1: 4}$ and F3 $3_{1: 8}$ showed a tremendous increase in solubility as compared to the other marketed products of tamoxifen $[38,25]$.

\section{Temozolomide}

Author investigated phenyl carbonate based $\beta$-CD NS for in vitro toxicity appraisal of temozolomide to become a potential drug for the treatment of glioma [40]. For the treatment of gliomas, this has been utilized as first-line therapy after its surgical resection [41]. They depict significant difficulties among which is the short halflife of $1.8 \mathrm{~h}$ and protein binding of $15 \%$, due to which it requires intermittent dosing [41,37]. Site targeting of temozolomide has become successful by utilizing nanotechnology [38,41-48]. Using magnetic resonance spectroscopy, researches affirmed the structure of NSs. They assessed the drug interaction, wherein a slight shift in the stated wavelength of the molecule prompt interaction of water-hating groups. The complexation and embodiment inside NSs were done using Fourier- transform infrared (FTIR), differential scanning calorimetry 
(DSC), and X-ray diffraction (XRD), as the drug peaks were either moved or concealed after formulation with NS. The slow in vitro release was achieved in case of NSs based formulation of temozolomide. Toxicity of the NSs formulations was equivalent to that of free drug, changes were seen in the morphology of human glioblastoma astrocytoma extracted from a malignant tumor, which was twisted or deteriorated after the procedure. The formulations developed showed a potential delivery to achieve the site targeting of the brain [36].

\section{Curcumin}

Curcumin is a hydrophobic polyphenolic phytochemical which has poor liquid solubility at acidic and unbiased $\mathrm{pH}$, yet is dissolvable at basic $\mathrm{pH}$ [49]. It is a critical constituent of turmeric powder. Other than being a solid cancer preventive agent, it also acts as a cardioprotective, neuroprotective, calming, and antiatherosclerotic specialist (a nonexclusive rundown). Curcumin has been comprehensively declared as a propitious anticancer agent $[44,50]$. A few examinations suggest that curcumin may be a propitious medicine for the treatment of different sorts of tumors such as a bosom disease, colon threatening development, kidney harm, leukemia, hepatocellular carcinoma, and prostate dangerous development [46]. Curcumin applies its antineoplastic properties through its consequences on atomic nuclear factor- $\mathrm{\kappa B}$, tumor necrosis factor- $\alpha$, interleukins, interferon $\gamma$, c-Jun N-terminal kinase, cyclooxygenases, mammalian focal point of mechanistic target of rapamycin, protein kinase $\mathrm{C}$, mitogen-activated protein kinase, peroxisome proliferator-started receptory, etc. $[44,45]$. It prevents cell proliferation and metastasis and at the same time, induces apoptosis by modifying these previously mentioned pathways. It has likewise been hypothesized that because of its pleiotropic properties, curcumin could also be more powerful than a solitary pathway focused on therapy [45]. Despite its wide range of uses, it benches to bedside transition afflicted with plenty of formulation challenges [47-51]. It is evident that curcumin shows solubility related to lower gastrointestinal absorption and lower oral bioavailability and experiences significant metabolism [44]. It degrades at physiological $\mathrm{pH}$ values [50]. Significant formulation efforts have been made to improve these issues of curcumin delivery [47].

Kurien et al. [52] have developed $\beta$-CD NSs-curcumin formulations as an effective antineoplastic drug alternative. The scientists showed multiple times enhancement in solubility of curcumin compared to plain curcumin and around multiple times when contrasted to the $\beta-C D$ complex of curcumin. The conjecture is that the higher solvency is due to complexation of curcumin with NSs and furthermore crosslinking of $\mathrm{CD}$ gives an appropriate environment for drug atoms to situate. The formulation had a normal molecule size of around $487 \mathrm{~nm}$ and performance index (PI) of 0.476 demonstrating a unimodal molecular size distribution with a narrow range. Its zeta potential qualities were about $-27 \mathrm{mV}$, thought adequately high for the development of a steady suspension. Significant peaks of curcumin that was obtained were entirely concealed or moved, and a portion of the NSs peaks moved indicating interactions at an atomic level. The researchers also assumed that as the drug in an amorphous state can easily diffuse through the polymeric NSs matrix giving a controlled release. In DSC, the peak of curcumin at $176^{\circ} \mathrm{C}$ was not acquired indicating that it formed an inclusion complex. In vitro sustained release of curcumin was studied by researchers over a period of 2 days. Up to the concentration of $2 \mathrm{mg} / \mathrm{ml}$, the formulation was found to be non-hemolytic. Cytotoxicity studies carried on MCF- 7 cells utilizing 3-(4,5-dimethylthiazol-2-yl)- 2,5-diphenyltetrazolium bromide assay demonstrated that curcumin formulation has a similar virulent quality to curcumin, in this way recommending the sub-atomic structure stays unaltered after the formulation [52].

\section{Resveratrol}

"Resveratrol is a stilbenoid, natural phenol is generally present in daily diet intake, for example, grapes, groundnut, pistachios, and blueberries. It is generally acknowledged as a potential antioxidant, antioncogenic property, and reduces inflammation [54]. Regardless of the plenty of survey information accessible and wide scope of medical importance, the clinical utilization of resveratrol is restricted by its short halflife [55] and immense and fast digestion pursued by excretion $[54,56]$. In addition, research work has shown that bioavailability of this drug taken orally is extremely insignificant therefore is a death blow for its further clinical research $[54,57,59]$. Regardless of the extensive research, the information on the drug plasma level still stays dim [59]. A few activities have been going onto overcome the limitation of resveratrol using several methods such as nanoparticle technology, niosomes, and nanovesicles [60-66]. Researcher utilized NSs to enhance the solvency, soundness, and skin penetrability of resveratrol [67]. The modified two formulations of NSs are prepared with different concentrations of crosslinkers (1:2 and 1:4, the molar premise of CD: CDI). Solvency of resveratrol was enhanced by around 33 times (F1:2) and by around 48 times (F1:4) when compared to the marketed drug. The significant changes in the FTIR extending peaks in NS recommended interaction of NS with resveratrol. Furthermore, DSC and XRD, additionally, confirmed the perception of FTIR wherein the endotherm showed reduced intensity, while the long-range crystalline peaks of resveratrol in X-beam range changed definitely to expansive peaks recommending amorphous nature. Resveratrol release was more uniform and complete from the F1:4 formulations compared to F1:2. Proposing improved photostability of resveratrol in formulation with NSs. Formulation F1:4 showed a higher level of cytotoxicity when compared with the drug in HCPC-I cells, which is both dose and time-dependent. A two-fold higher in vitro rabbit mucosa acquisition of resveratrol from F1:4 was seen by the researchers when contrasted with the drug dispersed in a hydroalcoholic blend $(1: 1 \mathrm{v} / \mathrm{v})$. They also perceived higher permeation of resveratrol across pig skin through the NSs formulation as compared to the drug dispersed in a hydroalcoholic mixture [68]."

\section{Quercetin}

"Quercetin is flavonoid that is found in vegetables, organic products, leaves, grains, and seeds, which possess a great advantage as an anticancerous agent [74]. The dynamic type of quercetin is an aglycone frame displaying exceptionally poor oral bioavailability ( $>2 \%$ in people), which indicates low solubility and possess difficulty in disintegration in the gastrointestinal tract $[69,70]$. Moreover, it additionally experiences broad first-pass metabolism, which moreover limits its oral delivery [71]. Quercetin belongs to BCS Class II, i.e. have low solubility $(7.7 \mu \mathrm{g} / \mathrm{mL})$, which was accounted to be upgraded using nanoparticles but failed to produce remarkable results by increasing solubility to just about $0.4 \mathrm{mg} / \mathrm{mL}[71,72]$. Hence, there is a requirement for further enhancing the physicochemical properties of quercetin [71]. Few novel advancements used by specialists to defeat the issues related to quercetin delivery are SNEDDS, biodegradable nanoparticles, polymeric microparticulate frameworks, nanocrystals, lipid nanoemulsions, etc. [73]."

"Anandam and Selvamuthukumar investigated NSs with diphenyl carbonate for enhancing the quercetin delivery [74]. They arranged five distinct sorts of quercetin NSs (using NSs with a various crosslinking concentration of CD and diphenyl carbonate) 1:2-1:10, molar proportions for their examinations. Solvency of quercetin was upgraded by around 20 overlays compared to the drug. With about $40-50 \% \mathrm{w} / \mathrm{w}$ stacking, F1:4 and F1:6 emerged as the ideal formulae. Peak moves and peak expanding in the FTIR spectra showed a unique finger impression region of quercetin after forming NSs recommended interactions at atomic dimensions. Further, utilizing Raman spectroscopy, the creators saw that essential markers of quercetin have significantly concealed or moved after complexation with NSs, which straightforwardly validated their FTIR findings. Further DSC and XRD discoveries support complexation of the molecule with NSs. Particular crests in XRD spectra were covered after forming of NSs, proposing the amorphization of the drug post complexation with NSs. As the examination showed a particular size division of NSs pretreated (mean size of NSs $<100 \mathrm{~nm}$ ), the molecule size was additionally observed to be in the scope of $41-94 \mathrm{~nm}$ which is less than the past reports by Trotta et al. [74-77,81,85]. The particles were round, consistently scattered, and confirmed a tight 
size delivery with a low PI. Zeta potential obtain was on the negative side. The NSs discharged quercetin in an altogether quicker rate of $91 \%-98 \%$ release up to $24 \mathrm{~h}$ when compared with the marketed drug. Over half of the drug degraded after $6 \mathrm{~h}$ in simulated intestinal liquid and $<10 \%$ drug degraded in NSs. On conducting photostability studies, about $22 \%$ of the pure drug got degraded on exposure to light, while $12 \%$ drug got degraded in NSs. Both the examinations showed better physicochemical properties and photostability of quercetin on forming NSs $[75,99] . "$

Further, in vitro release for quercetin NS and marketed formulation are performed to differentiate the release. The radical (2,2-diphenyl- 1-picrylhydrazyl) action which is the main parameter depicting cancer as a preventive agent potentially demonstrated that NS of quercetin showed around 500-850 overlay higher action compared to quercetin (F1:4: 569-crease, and F1:6: 824-overlap). Anti-superoxide arrangement test uncovered showed 500 times higher action of NS compared to quercetin (F1:4: 556-crease and F1:6: 723-overlap), while the superoxide anion-searching action painted a comparative picture wherein there was an upgrade of around 600-1200 crease action by NSs (F1:4: 612-overlap and F1:6: 1234-crease).

\section{NS Formulations to deliver oxygen}

NSs have capabilities of storing certain gases such as carbon dioxide, oxygen, and 1--methylcyclopropene which have a promising role in cosmeceuticals, pharmaceuticals, and biotechnology. Certain CD- based NSs have been synthesized using alpha, beta, or gamma with carbonyldiimidazole as oxygen delivering formulation. For the first time, Researchers explained the gas storing capacity and delivery of NSs as a potential apparatus in hypoxia-related to cancer. Deficiency of oxygen causes hypoxia with certain limits for the treatment. Patients with hypoxic cervical malignant growths survival rate have been poor [86]. Thinking about the capability of NSs in drug epitome and controlled discharge, Cavalli et al. orchestrated three distinct kinds of CDI based NS, i.e., alpha, beta, and gamma NS utilizing the particular CD particles. NS suspension was homogenized the high shear rate for about 2-3 min. Later it was sealed and was saturated with oxygen. This was stored at $25^{\circ} \mathrm{C}$ to evaluate the stability of NSs. A toxicity study was carried out using Vero cells. About $5 \mathrm{mg} / \mathrm{mL}$, NS fluid scattering was infused with saline in vials in hypoxic conditions at a steady temperature to study the in vitro $\mathrm{O}_{2}$ discharge design with an in-line oximeter. An in-house modified gadget was utilized to check the concentrate penetration of $\mathrm{O}_{2}$; wherein two compartments were isolated using a thin silicon membrane. The surface region esteems for NS were in the scope of 40 and $50 \mathrm{~m}^{2} / \mathrm{g}$. NS were circular with molecule sizes of 400 and $550 \mathrm{~nm}$ with restricted dissemination. Zeta potential was toward the negative side $(-30 \mathrm{mV})$. The $\mathrm{O}_{2}$ formulation did not show toxicity to Vero cells. The suspension did not show any agglomeration or degradation at $25^{\circ} \mathrm{C}$ when stored for 15 days. It was observed that ultrasound enhanced the $\mathrm{O}_{2}$ discharge by about $58 \%$ from NS formulation. Researchers compared two formulation one with oxygenated NSs and another with plain oxygen. Oxygen saturation NSs demonstrated that the $\beta$-CD NS detailing displayed a higher pervasion compared to their $\alpha$-and $\gamma$-CD partners. Oxygen penetration from the $\beta$-CD NS detailing enhanced by about $192 \%$ with the utilization of ultrasound showed underlying $\mathrm{O}_{2}$ spike.

The creators at that point investigated a Pluronic ${ }^{\circledR}$-based hydrogel arrangement of the $\beta$-CD NS, which gave a uniform, continued arrival of $\mathrm{O}_{2}$ without the spike (with or without ultrasound) [77]. Proceeding with the examination further, Trotta et al. used an adjusted philosophy for designing the $\mathrm{O}_{2}$-stacked NSs. They included sodium chloride, PEG 400 , and decafluoropentane in the blend alongside NSs and water to additionally enhance the $\mathrm{O}_{2}$ stacking, stockpiling, and delivery. They built two formulations of $\beta$-CD NSs and one of $\alpha$-CD NSs. The physical attributes acquired were like the past examination. These details were nonhemolytic and safe to be infused in vivo as assessed by in vitro hemolytic action. Supported $\mathrm{O}_{2}$ discharge for up to $60 \mathrm{~min}$ was gotten by NS details. Ultrasound positively affected $\mathrm{O}_{2}$ discharge.
A Pluronic ${ }^{\circledR}$ - based hydrogel prompted the bring down of $\mathrm{O}_{2}$ discharge. Ultrasound achieved a $30 \%$ increase in the penetration rate of $\mathrm{O}_{2}$ from the $\beta$-CD NSs formulation [79].

\section{ETB glutathione}

ETB glutathione is used to treat lung cancer, ovarian, head, and neck as well. However, due to the several limitations which include poor bioavailability, unstable at the acidic environment and first-pass metabolism making it difficult to deliver the drug to the targeted site. Furthermore, it possesses several side effects such as skin rashes, anemia, Stevens-Johnson syndrome. "Momin et al. explored the efficacy of ETB glutathione that has increased by incorporating it into the nanocarrier systems. Among which the most useful technology for site target drug delivery system was NSs without showing any cytotoxicity on several cancer cell lines. They incorporated glutathione into NSs for a single step reaction at room temperature. Evaluation of in vitro release was carried out using high-performance liquid chromatography technique. Cytotoxicity was evaluated on human lung cancer (A549) cell lines and in vivo studies were carried out BALB mice. The obtained NSs were sphere sized $(212 \pm 245 \mathrm{~nm})$ with drug entrapment capacity of $92.34 \% \pm 5.31 \%(\mathrm{p}<0.001)$. Highest in vitro drug release was $76.89 \% \pm 0.1 \%$ release at $168 \mathrm{~h}$, which was proportional to the concentration of ETB glutathione demonstrating tumor targeting. It showed $97.5 \%$ hindrance in tumor development on regulating NSs when compared with plain ETB (48\% restraint) depicting that NSs directly targets the tumors site preventing unnecessary drug exposure to other cells [97].

\section{Delivery of water-soluble and sparingly solvent anticancer molecules}

Trotta, Cavalli, and collaborators assessed the properties of NSs to enhance water solubility and sparingly soluble anticancer particles, for example, doxorubicin, 5-FU $[80,81]$. Doxorubicin is a standout among the most usually utilized particles for treating tumors of the major organ and delicate tissues [82]. Doxorubicin hydrochloride infusion was the first liposomal anticancer item to get regulatory approval. In a long time, a great part of the examination endeavors have been engaged toward investigating nanotechnology devices for decreasing the cardiotoxicity and expanding the explicitness of doxorubicin [7790] Cavalli et al. used NSs out of the blue for regulating the arrival of doxorubicin [90,81]. Doxorubicin was stacked in NSs (stacking of $20 \% \mathrm{w} / \mathrm{w}$ ) as demonstrated by previously mentioned methods. The creators discovered that doxorubicin was discharged in a moderate and continued way after consolidation in NSs. In vitro, release contemplates recommended that doxorubicin was released in a $\mathrm{pH}$-depended manner at a moderate rate of about $1 \%$ at acidic $\mathrm{pH}$ after $2 \mathrm{~h}$. At basic $\mathrm{pH}$, doxorubicin discharge was about $29 \%$ after $3 \mathrm{~h}$. This indicates that NS protects doxorubicin from acidic environment, especially in the stomach. It shields the drug and releases it into basic condition, i.e., intestine and duodenum. Top to bottom examinations is expected to approve this specific discharge conduct conclusively $[87,88]$. Further, Researchers, enhanced the properties of 5 -FU by utilizing gamma CDbased NSs. 5-FU is the most favored drug in the treatment of colorectal disease, stomach malignancy, and cervical malignant growth. It is a very polar drug with pka estimations of 8.0-13.0 [91]. It has low solubility when taken orally. It displays low terminal half-life (8-20 min) by means of the parenteral route [91]. It causes serious side effects and is highly photosensitive when infused intravenously [91]. Research to relieve these issues of 5-FU delivery has used as imaginative methodologies, for example, gellan gum microbeads, chitosan polycarbophil inter polyelectrolyte complex, mastic gum-based frameworks, egg whites nanoparticles, strong lipid nanoparticles, and other traditional polymeric nanoparticles [83-96,98]."

\section{CONCLUSION}

CD-based NSs have shown promising results in cancer therapy. In spite of that few structural modifications required to enhance the efficacy of NSs which can also function through external or internal stimuli. New research 
has been carried out on cisplastine and doxorubicin. NS offers an extra favorable position of different associating destinations for medicating stacking. The crosslinking thickness and the blend can be tuned to tweak the drug discharge in light of the upgrades. In our underlying investigations, we gave promising outcomes a progression of disulfide-containing NS (with fluctuating measures of the delicate disulfide NS) nanosized by high weight homogenization (normal size $\sim 200 \mathrm{~nm}$ ). The research will likewise likely additionally proceed toward investigating different courses of the organization, for example, intraocular, intratumoral, topical, parenteral, buccal, what's more, and nasal-to-cerebrum.

\section{ACKNOWLEDGMENTS}

The authors express their gratitude to the JSS Academy of Higher Education and Research and JSS College of Pharmacy, Mysuru, for providing necessary support in due course of the work.

\section{AUTHOR'S CONTRIBUTIONS}

All the authors have contributed equally in the design, development, review and finalization of the contents of the manuscript.

\section{CONFLICTS OF INTEREST}

The authors confirm that this article content has no conflicts of interest.

\section{REFERENCES}

1. Osmani RA, Aloorkar NH, Kulkarni AS, Kulkarni PK, Hani U, Thirumaleshwar S, et al. Novel cream containing microsponges of anti- acne agent: Formulation development and evaluation. Curr Drug Deliv 2015;12:504-16.

2. Rosen M. Delivery system handbook for personal care and cosmetic products: Technology, applications and formulations, personal care and cosmetic technology. Norwich: William Andrew. 2005.

3. Swaminathan S, Cavalli R, Trotta F. Cyclodextrin-based nanosponges: A versatile platform for cancer nanotherapeutics development. Wiley Interdiscip Rev Nanomed Nanobiotechnol 2016;8:579-601

4. Pushpalatha R, Selvamuthukumar S, Kilimozhi D. Nanocarrier mediated combination drug delivery for chemotherapy - A review. J Drug Deliv Sci Technol 2017;39:362-71.

5. Momin MM, Zaheer Z, Zainuddin R, Sangshetti JN. Extended release delivery of erlotinib glutathione nanosponge for targeting lung cancer. Artif Cells Nanomed Biotechnol 2018;46:1064-75.

6. Luo Y, Prestwich GD. Cancer-targeted polymeric drugs. Curr Cancer Drug Targets 2002;2:209-26.

7. Nie S, Xing Y, Kim GJ, Simons JW. Nanotechnology applications in cancer. Annu Rev Biomed Eng 2007;9:257-88.

8. Fojo T, Bates S. Strategies for reversing drug resistance. Oncogene 2003;22:7512-23.

9. Tsuruo T, Naito M, Tomida A, Fujita N, Mashima T, Sakamoto H, et al. Molecular targeting therapy of cancer: Drug resistance, apoptosis and survival signal. Cancer Sci 2003;94:15-21.

10. Couvreur P. Nanoparticles in drug delivery: Past, present and future. Adv Drug Deliv Rev 2013;65:21-3.

11. Couvreur P, Tulkens P, Roland M, Trouet A, Speiser P. Nanocapsules: A new type of lysosomotropic carrier. FEBS Lett 1977;84:323-6.

12. Chen ZG. Small-molecule delivery by nanoparticles for anticancer therapy. Trends Mol Med 2010;16:594-602.

13. Matsumura Y, Maeda H. A new concept for macromolecular therapeutics in cancer chemotherapy: Mechanism of tumoritropic accumulation of proteins and the antitumor agent smancs. Cancer Res 1986;46:6387-92.

14. Lammers T, Kiessling F, Hennink WE, Storm G. Drug targeting to tumors: Principles, pitfalls and (pre-) clinical progress. J Control Release 2012;161:175-87.

15. Maeda H, Wu J, Sawa T, Matsumura Y, Hori K. Tumor vascular permeability and the EPR effect in macromolecular therapeutics: A review. J Control Release 2000;65:271-84.

16. Maeda H, Nakamura H, Fang J. The EPR effect for macromolecular drug delivery to solid tumors: Improvement of tumor uptake, lowering of systemic toxicity, and distinct tumor imaging in vivo. Adv Drug Deliv Rev 2013;65:71-9.

17. Decuzzi P, Godin B, Tanaka T, Lee SY, Chiappini C, Liu X, et al. Size and shape effects in the biodistribution of intravascularly injected particles. J Control Release 2010;141:320-7.
18. Nurgali K, Jagoe RT, Abalo R. Adverse effects of cancer chemotherapy: Anything new to improve tolerance and reduce sequelae?. Frontiers in pharmacology 2018;22:245.

19. Desai N, Trieu V, Yao Z, Louie L, Ci S, Yang A, et al. Increased antitumor activity, intratumor paclitaxel concentrations, and endothelial cell transport of cremophor-free, albumin-bound paclitaxel, ABI- 007, compared with cremophor-based paclitaxel. Clin Cancer Res 2006; $12: 1317-24$.

20. Trotta F, Caldera F, Dianzani C, Argenziano M, Barrera G, Cavalli R. Glutathione bioresponsive cyclodextrin nanosponges. Chem Plus Chem 2016;81:439-43.

21. Lee CL, Wu CC, Chiou H, Syu C, Huang C, Yang C. Mesopourous platinum nanosponges as electrocatalysis for the oxygen reduction reaction in an acidic electrolyte. Int $\mathrm{J}$ Hydrogen Energy 2011;36:6433- 40

22. Jordan VC. Tamoxifen (ICI46,474) as a targeted therapy to treat and prevent breast cancer. Br J Pharmacol 2006;147 Suppl 1:S269-76.

23. Buddhadev L, Biswajit M.Tamoxifen citrate encapsulated sustained release liposomes: Preparation and evaluation of physicochemical properties. Sci Pharm 2010;78:507-15.

24. Jayanta KS, Rita M, Saibal KB, Ranadeep M, Angshuman B. Controlled release of tamoxifen citrate encapsulated in cross-linked guar gum nanoparticles. Int J Biol Macromol 2011;49:390-6.

25. Torne S, Darandale S, Vavia P, Trotta F, Cavalli R. Cyclodextrin- based nanosponges: Effective nanocarrier for tamoxifen delivery. Pharm Dev Technol 2013;18:619-25.

26. Martínez A, Benito-Miguel M, Iglesias I, Teijón JM, Blanco MD. Tamoxifen-loaded thiolated alginate-albumin nanoparticles as antitumoral drug delivery systems. J Biomed Mater Res A 2012;100:1467-76.

27. Bhatia A, Singh B, Raza K, Shukla A, Amarji B, Katare OP. Tamoxifenloaded novel liposomal formulations: Evaluation of anticancer activity on DMBA-TPA induced mouse skin carcinogenesis. J Drug Target 2012;20:544-50.

28. Bilensoy E. Amphiphilic cyclodextrin nanoparticles for effective and safe delivery of anticancer drugs. Adv Exp Med Biol 2015;822:201.

29. Barbieri S, Sonvico F, Como C, Colombo G, Zani F, Buttini F, et al. Lecithin/chitosan controlled release nanopreparations of tamoxifen citrate: Loading, enzyme-trigger release and cell uptake. J Control Release 2013;167:276-83.

30. Sarwa KK, Suresh PK, Debnath M, Ahmad MZ. Tamoxifen citrate loaded ethosomes for transdermal drug delivery system: Preparation and characterization. Curr Drug Deliv 2013;10:466-76.

31. Jain AS, Goel PN, Shah SM, Dhawan VV, Nikam Y, Gude RP, et al. Tamoxifen guided liposomes for targeting encapsulated anticancer agent to estrogen receptor positive breast cancer cells: In vitro and in vivo evaluation. Biomed Pharmacother 2014;68:429-38.

32. Pandey SK, Ghosh S, Maiti P, Haldar C. Therapeutic efficacy and toxicity of tamoxifen loaded PLA nanoparticles for breast cancer. Int J Biol Macromol 2015;72:309-19.

33. Vivek R, Nipun Babu V, Thangam R, Subramanian KS, Kannan S. PH- responsive drug delivery of chitosan nanoparticles as tamoxifen carriers for effective anti-tumor activity in breast cancer cells. Colloids Surf B Biointerfaces 2013;111:117-23.

34. How CW, Rasedee A, Manickam S, Rosli R. Tamoxifen-loaded nanostructured lipid carrier as a drug delivery system: Characterization, stability assessment and cytotoxicity. Colloids Surf B Biointerfaces 2013;112:393-9.

35. Trotta F, Dianzani C, Caldera F, Mognetti B, Cavalli R. The application of nanosponges to cancer drug delivery. Expert Opin Drug Deliv 2014;11:931-41.

36. Jain D, Gursalkar T, Bajaj A. Nanosponges of an anticancer agent for potential treatment of brain tumors. Am J Neuroprot Neuroregen 2013;5:32-43

37. Newlands ES, Stevens MF, Wedge SR, Wheelhouse RT, Brock C. Temozolomide: A review of its discovery, chemical properties, pre- clinical development and clinical trials. Cancer Treat Rev 1997;23:35-61.

38. Chen Z, Lai X, Song S, Zhu X, Zhu J. Nanostructured lipid carriers based temozolomide and gene co-encapsulated nanomedicine for gliomatosis cerebri combination therapy. Drug Deliv 2016;23:1369-73.

39. Dilnawaz F, Sahoo SK. Enhanced accumulation of curcumin and temozolomide loaded magnetic nanoparticles executes profound cytotoxic effect in glioblastoma spheroid model. Eur J Pharm Biopharm 2013;85:452-62.

40. Di Martino A, Sedlarik V. Amphiphilic chitosan-grafted-functionalized polylactic acid based nanoparticles as a delivery system for doxorubicin and temozolomide co-therapy. Int J Pharm 2014;474:134-45. 
41. Dou M, Huang G, Xi Y, Zhang N. Orthogonal experiments for optimizing the formulation and preparation conditions of temozolomide solid lipid nanoparticles. Sheng Wu Yi Xue Gong Cheng Xue Za Zhi 2008;25:1141-5.

42. Jain A, Jain SK. Formulation and optimization of temozolomide nanoparticles by 3 factor 2 level factorial design. Biomatter 2013;3. pii: e25102.

43. Thirupathy A, Srinivas P, Ravindhra Babu DS, Mamidi S. Formulation and evaluation of sustained release implantable microspheres of temozolomide for brain targeting prepared by a novel technique. Int J Pharm Pharm Sci 2011;3:187-94.

44. Beevers CS, Huang S. Pharmacological and clinical properties of curcumin. Botanics 2011;1:5-18.

45. Naksuriya O, Okonogi S, Schiffelers RM, Hennink WE. Curcumin nanoformulations: A review of pharmaceutical properties and preclinical studies and clinical data related to cancer treatment. Biomaterials 2014;35:3365-83

46. Crupi V, Majolino D, Mele A, Rossi B, Trotta F, Venuti V. Modelling the interplay between covalent and physical interactions in cyclodextrin- based hydrogel: Effect of water confinement. Soft Matter 2013;9:6457-64

47. Prasad S, Tyagi AK, Aggarwal BB. Recent developments in delivery, bioavailability, absorption and metabolism of curcumin: The golden pigment from golden spice. Cancer Res Treat 2014;46:2-18.

48. Anand P, Kunnumakkara AB, Newman RA, Aggarwal BB. Bioavailability of curcumin: Problems and promises. Mol Pharm 2007;4:807-18.

49. Anand P, Sundaram C, Jhurani S, Kunnumakkara AB, Aggarwal BB. Curcumin and cancer: An "old-age" disease with an "age-old" solution. Cancer Lett 2008;267:133-64.

50. Wang YJ, Pan MH, Cheng AL, Lin LI, Ho YS, Hsieh CY, et al. Stability of curcumin in buffer solutions and characterization of its degradation products. J Pharm Biomed Anal 1997;15:1867-76.

51. Tønnesen HH, Másson M, Loftsson T. Studies of curcumin and curcuminoids. XXVII. Cyclodextrin complexation: Solubility, chemical and photochemical stability. Int J Pharm 2002;244:127-35.

52. Kurien BT, Singh A, Matsumoto H, Scofield RH. Improving the solubility and pharmacological efficacy of curcumin by heat treatment. Assay Drug Dev Technol 2007;5:567-76.

53. Darandale SS, Vavia PR. Cyclodextrin-based nanosponges of curcumin: Formulation and physicochemical characterization. J Incl Phenom Macrocycl Chem 2013;75:315-22.

54. Amri A, Chaumeil JC, Sfar S, Charrueau C. Administration of resveratrol: What formulation solutions to bioavailability limitations? J Control Release 2012;158:182-93.

55. Vian MA, Tomao V, Gallet S, Coulomb PO, Lacombe JM. Simple and rapid method for cis- and trans-resveratrol and piceid isomers determination in wine by high-performance liquid chromatography using chromolith columns. J Chromatogr A 2005;1085:224-9.

56. Baur JA, Sinclair DA. Therapeutic potential of resveratrol: The in vivo evidence. Nat Rev Drug Discov 2006;5:493-506.

57. Wenzel E, Somoza V. Metabolism and bioavailability of transresveratrol. Mol Nutr Food Res 2005;49:472-81.

58. Walle T, Hsieh F, DeLegge MH, Oatis JE Jr., Walle UK. High absorption but very low bioavailability of oral resveratrol in humans. Drug Metab Dispos 2004;32:1377-82.

59. Cottart CH, Nivet-Antoine V, Laguillier-Morizot C, Beaudeux JL. Resveratrol bioavailability and toxicity in humans. Mol Nutr Food Res 2010;54:7-16.

60. Lu Z, Cheng B, Hu Y, Zhang Y, Zou G. Complexation of resveratrol with cyclodextrins: Solubility and antioxidant activity. Food Chem 2009; 113:17-20.

61. Amri A, Le Clanche S, Thérond P, Bonnefont-Rousselot D, Borderie D, Lai-Kuen R, et al. Research paper: Resveratrol selfemulsifying system increases the uptake by endothelial cells and improves protection against oxidative stress-mediated death. Eur J Pharm Biopharm 2014;86:418-26.

62. Friedrich RB, Kann B, Coradini K, Offerhaus HL, Beck RCR, Windbergs M. Skin penetration behaviour of lipid-core nanocapsules for simultaneous delivery of resveratrol and curcumin. Eur J Pharm Sci 2015;78:204-13

63. Teskac K, Kristl J. The evidence for solid lipid nanoparticles mediated cell uptake of resveratrol. Int J Pharm 2010;390:61-9.

64. Caddeo C, Manconi M, Fadda AM, Lai F, Lampis S, Diez-Sales O, et al. Nanocarriers for antioxidant resveratrol: Formulation approach, vesicle self-assembly and stability evaluation. Colloids Surf B Biointerfaces 2013;111:327-32.

65. Chauhan AS. Dendrimer nanotechnology for enhanced formulation and controlled delivery of resveratrol. Ann N Y Acad Sci 2015;1348:134- 40.

66. Pando D, Matos M, Gutiérrez G, Pazos C. Formulation of resveratrol entrapped niosomes for topical use. Colloids Surf B Biointerfaces 2015;128:398-404.

67. Jagtap S, Meganathan K, Wagh V, Winkler J, Hescheler J, Sachinidis A. Chemoprotective mechanism of the natural compounds, epigallocatechin-3-O-gallate, quercetin and curcumin against cancer and cardiovascular diseases. Curr Med Chem 2009;16:1451-62.

68. Ansari KA, Vavia PR, Trotta F, Cavalli R. Cyclodextrin-based nanosponges for delivery of resveratrol: In vitro characterisation, stability, cytotoxicity and permeation study. AAPS PharmSciTech 2011;12:279-86.

69. Wang Q, Bao Y, Ahire J, Chao Y. Co-encapsulation of biodegradable nanoparticles with silicon quantum dots and quercetin for monitored delivery. Adv Healthc Mater 2013;2:459-66.

70. Kumari A, Yadav SK, Pakade YB, Singh B, Yadav SC. Development of biodegradable nanoparticles for delivery of quercetin. Colloids Surf B Biointerfaces 2010;80:184-92.

71. Tran TH, Guo Y, Song D, Bruno RS, Lu X. Quercetin-containing self-nanoemulsifying drug delivery system for improving oral bioavailability. J Pharm Sci 2014;103:840-52.

72. Gao L, Liu G, Wang X, Liu F, Xu Y, Ma J. Preparation of a chemically stable quercetin formulation using nanosuspension technology. Int J Pharm 2011;404:231-7.

73. Cavalli R, Trotta F, Tumiatti W. Cyclodextrin-based nanosponges for drug delivery. J Incl Phenom Macrocycl Chem 2006;56:209-13.

74. Anandam S, Selvamuthukumar S. Fabrication of cyclodextrin nanosponges for quercetin delivery: Physicochemical characterization, photostability, and antioxidant effects. J Mater Sci 2014;49:8140-53.

75. Lembo D, Swaminathan S, Donalisio M, Civra A, Pastero L, Aquilano D, et al. Encapsulation of acyclovir in new carboxylated cyclodextrin- based nanosponges improves the agent's antiviral efficacy. Int J Pharm 2013;443:262-72.

76. Swaminathan S, Pastero L, Serpe L, Trotta F, Vavia P, Aquilano D, et al. Cyclodextrin-based nanosponges encapsulating camptothecin: Physicochemical characterization, stability and cytotoxicity. Eur J Pharm Biopharm 2010;74:193-201.

77. Swaminathan S, Vavia PR, Trotta F, Cavalli R. Nanosponges encapsulating dexamethasone for ocular delivery: Formulation design, physicochemical characterization, safety and corneal permeability assessment. J Biomed Nanotechnol 2013;9:998-1007.

78. Cavalli R, Akhter AK, Bisazza A, Giustetto P, Trotta F, Vavia P. Nanosponge formulations as oxygen delivery systems. Int $\mathrm{J}$ Pharm 2010;402:254-7.

79. Trotta F, Cavalli R, Martina K, Biasizzo M, Vitillo J, Bordiga S, et al. Cyclodextrin nanosponges as effective gas carriers. J Incl Phenom Macrocycl Chem 2011;71:189-94.

80. Trotta F, Zanetti M, Cavalli R. Cyclodextrin-based nanosponges as drug carriers. Beilstein J Org Chem 2012;8:2091-9.

81. Cavalli R, Trotta F, Tumiatti W, Serpe L, Zara GP. 5-Fluorouracil loaded $\beta$-cyclodextrin nanosponges: In vitro characterization and cytotoxicity. In: Proceedings XIII International Cyclodextrin Symposium, Turin, Italy; 2006. p. 207

82. Rivankar S. An overview of doxorubicin formulations in cancer therapy. J Cancer Res Ther 2014;10:853-8.

83. Bhaskar C, Ahmed F, Kondapi AK, Golla K. A target-specific oral formulation of doxorubicinprotein nanoparticles: Efficacy and safety in hepatocellular cancer. J Cancer Educ 2013;4:644-52.

84. Mura P, Bragagni M, Mennini N, Ghelardini C. Development and characterization of niosomal formulations of doxorubicin aimed at brain targeting. J Pharm Pharm Sci 2012;15:184-196.

85. Candido CD, Campos ML, Correa Vidigal Assumpção JU, Pestana KC, Padilha EC, Carlos IZ, et al. Biocompatible microemulsion modifies the tissue distribution of doxorubicin. J Pharm Sci 2014;103:3297-301.

86. Elbialy NS, Fathy MM, Khalil WM. Preparation and characterization of magnetic gold nanoparticles to be used as doxorubicin nanocarriers. Phys Med 2014;30:843-8.

87. Jiang SP, He SN, Li YL, Feng DL, Lu XY, Du YZ, et al. Preparation and characteristics of lipid nanoemulsion formulations loaded with doxorubicin. Int J Nanomedicine 2013;8:3141-50

88. Ryan GM, Kaminskas LM, Bulitta JB, McIntosh MP, Owen DJ, Porter CJ. PEGylated polylysine dendrimers increase lymphatic exposure to doxorubicin when compared to PEGylated liposomal and solution formulations of doxorubicin. $\mathrm{J}$ Control Release 2013;172:128-36

89. Win KY, Teng CP, Ye E, Low M, Han MY. Evaluation of polymeric nanoparticle formulations by effective imaging and quantitation 
of cellular uptake for controlled delivery of doxorubicin. Small 2015;11:1197-204

90. Levacheva I, Samsonova O, Tazina E, Beck-Broichsitter M, Levachev S, Strehlow B, et al. Optimized thermosensitive liposomes for selective doxorubicin delivery: Formulation development, quality analysis and bioactivity proof. Colloids Surf B Biointerfaces 2014:121:248-56

91. Mohamed SP, Pramod KT. Development and characterization of chitosan-polycarbophil interpolyelectrolyte complex-based 5-flurouracil formulations for buccal, vaginal and rectal application. DARU 2012;20:67:1-11.

92. Yassin AE, Anwer MK, Mowafy HA, El-Bagory IM, Bayomi MA, Alsarra IA. Optimization of 5-flurouracil solid-lipid nanoparticles: A preliminary study to treat colon cancer. Int J Med Sci 2010;7:398-408

93. Wilson B, Ambika TV, Patel RD, Jenita JL, Priyadarshini SR. Nanoparticles based on albumin: Preparation, characterization and the use for 5-flurouracil delivery. Int J Biol Macromol 2012;51:874-8.

94. Mallamma T, Thippeswamy BS, Bharathi DR, Snehalatha, Nagaraja TS,
Yogananda R, et al. Formulation and evaluation of 5-flurouracil loaded HSA nanoparticle for controlled drug delivery. Int J Adv Res 2013;7:23-30.

95. Nasr M, Saad IE. Formulation and evaluation of mastic gum as a compression coat for colonic delivery of 5-flurouracil. Int J Drug Deliv 2011;3:481-91

96. Sahoo SK, Sahoo SK, Behera A, Patil SV, Panda SK. Formulation, in vitro drug release study and anticancer activity of 5-fluorouracil loaded gellan gum microbeads. Acta Pol Pharm 2013;70:123-7.

97. Momin MM, Zaheer Z, Zainuddin R, Sangshetti JN. Extended release delivery of erlotinib glutathione nanosponge for targeting lung cancer. Artif Cells Nanomed Biotechnol 2018;46:1064-75.

98. Atil SS, Gupta VR, Gupta KS, Doddayya H. Effect of ph, selected cyclodextrins and complexation methods on the solubility of lornoxicam. Int J Pharm Pharm Sci 2014;6:324-7.

99. Sambandam B, Sathesh Kumar S, Ayyaswamy A, Nagarjuna Yadav BV, Thiyagarajan D. Synthesis and characterization of poly d-1 lactide (pla) nanoparticles for the delivery of quercetin. Int J Pharm Pharm Sci 2015;7:42-9. 